\title{
兴奋毒性损伤尾壳核增强 B-50(GAP-43) mRNA 的表达
}

\author{
张世仪 锁志明 张艳萍 * 刘 洁 \\ (中国医学科学院基础医学研究所, 中国协和医科大学基础医学院, 北京 100005)
}

\section{关键词 B-50(GAP-43)mRNA 尾壳核 兴奋每性损伤 原位杂交 大鼠}

长期以来, 人们一直认为成年哺乳动物中枢神经系统损伤后不能再生. 然而近数十年来 这一观点正在改变. 许多证据表明中枢神经损伤后有轴突侧支发芽或突触增生现象, 同时在 一定条件下其轴突能够再生 ${ }^{[1]}$. 可是对这些过程的分子机理仍很不了解. B-50 是一种重要 的神经生长相关蛋白 (亦命名为 GAP - 43, F1, neuromodulin, pp46). 已有资料说明 B-50 的 表达与发育期轴突生长或外周神经再生密切相关 ${ }^{[2]}$, 但有关其在中枢神经系统损伤后表达的 变化和意义尚不清楚. 本工作以兴奋毒性损伤大鼠的尾壳核 (Caudate putamen nucleus, CPN), 作为中枢神经系统损伤的一种模式 (也是 Huntington 氏病动物模型), 用地高辛配基 (Digoxigenin-dUTP) 标记 cDNA 探针的原位杂交方法, 检测损伤后 CPN 内 B-50mRNA 表 达的动态变化.

\section{1 材料和方法}

\section{1 动物和手术}

24 只雄性 Wistar 大鼠 (3 月龄) 在 $2.5 \%$ 三溴乙醇 (avertin, Merck 产品, $1.2 \mathrm{~mL} / 100 \mathrm{~g}$ ) 麻 醉下, 施行 CPN 内海人酸 (Kanic acid, KA, Sigma 产品) 注射. 按 Pellegrino 等的定位图 谱, 注射部位为 $\mathrm{A}=2.0, \mathrm{~L}=2.6, \mathrm{H}=4.0$. 动物分为双侧和单侧注射两组, 每组 12 只. 每侧 $\mathrm{KA}$ 用量为 $0.3 \mu \mathrm{g} / 0.5 \mu \mathrm{L}$. 单侧损伤组对照侧注射等体积生理盐水. 术后 $3 \mathrm{~d}$ 内有 4 只鼠死亡, 所 余 20 只于术后 $1 、 3 、 6$ 周时处死 (两组 3 个时间点的动物数均为 3,5 和 2 , 另有 3 只正常大鼠作 对照). 取出脑, 于液氮中贮存.

\section{2 组织制备}

取脑的 CPN 和海马区冰冻切片 $20 \mu \mathrm{m}$ 厚. 载玻片用 EAA 及三乙醇胺 (pH8.0) 缓冲液预 处理. 用 $4 \%$ 多聚甲醛 -PBS 缓冲液进行后固定. 切片浸在 $70 \%$ 乙醇中, 于 $-20{ }^{\circ} \mathrm{C}$ 贮存.

\section{3 尼氏 (Nissl) 染色}

手术后 3 周的 CPN 切片, 用 $1 \%$ 的甲苯胺兰进行常规染色，以确定损伤部位.

\section{4 探针制备}

用荷兰 Verhaagen 博士惠赠的质粒 pGBo(含大鼠 B-50 cDNA 插人片断 ), 转染 JM-109

1995-02-02 收稿, 1995-07-15 收修改稿

* 现在单位: 北京大学生物系, 北京 100871 
大肠杆菌. 大量扩增后回收并纯化 pGBo, 再用 PruII 酶切. 回收其中 B-50cDNA 之 204 碱基 对编码序列作为探针.

\section{5 原位杂交}

按照非放射性 DNA 标记及检测试剂盒 (Boehringer Mannbeim 公司) 介绍的方法, 以地 高辛配基标记探针。切片在杂交缓冲液 $(4 \times \mathrm{SSC}, 50 \%$ 甲酰胺, $1 \times$ Denhardt, $5 \%$ 硫酸葡聚糖，

$0.5 \mathrm{mg} / \mathrm{mL}$ 鲑精 DNA, $0.25 \mathrm{mg} / \mathrm{mL}$ 酵母 tRNA) 中于室温预杂交 $1 \mathrm{~h}$, 然后用含有

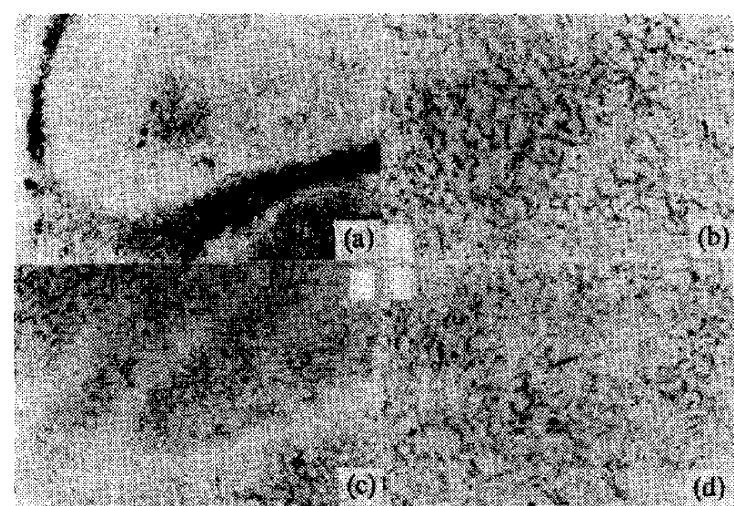

图 1 原位杂交检测 B-50 mRNA 的方法对照 (a) 阳性对照 (背侧海马), (b) 阴性对照: 用 RNA 酶预处理 (背侧海马), (c) 阴性对照: 用 RNA 酶预处理 (尾壳核), (d) 阴性对照: 不加抗体 - 酶复合物 (尾壳核) 变性探针 $(25 \mathrm{ng} / \mathrm{mL})$ 的新鲜杂交缓冲液取 代预杂交液, 于 $37^{\circ} \mathrm{C}$ 杂交过夜. 用 SSC 洗 片后进行免疫检测。即以抗地高辛配基的抗 体和碱性磷酸酶复合物与探针结合, 从而 使脑片中与探针杂交的 B-50 mRNA, 被 碱性磷酸酶催化的颜色反应显示出来。

方法对照: (1) 阳性对照以正常中枢神 经系统中 B-50 mRNA 高表达的海马切片进 行杂交(图 1(a)). (2) 阴性对照两项, 即在 杂交前用 RNA 酶消化所有 RNA 信号 (图 1(b),(c)) 或在免疫检测时不加抗体 酶复合物 (图 1(d)).

\section{6 半定量分析}

每组切片中具有代表性的结果拍摄成 照片后, 选每张照片中阳性反应最强的 6 个点, 通过 CS-910 型薄层扫描仪扫描. 以吸光度 (absorbance) 的峰值作为指标, 并作 统计学处理 $(t$ 检验).

\section{2 结果}

\section{$2.1 \mathrm{KA}$ 损伤 CPN 的组织学表现}

$\mathrm{KA}$ 注射后 3 周的脑切片上, 可见背侧 CPN 内有一约 $200 \sim 500 \mu \mathrm{m}$ 直径的神经元缺失

区. 而对照大鼠的 CPN 未见变化 (图 2).

\subsection{B-50mRNA 在成年 CPN 和海马内的表达}

在正常对照大鼠的双侧 CPN, 都出现弥散 性的 B-50mRNA 弱阳性标记信号 (图 3 (d)). 海马雉体细胞层有较强的阳性标记反应(图 1(a))

\section{$2.3 \mathrm{KA}$ 损伤 CPN 后 B-50mRNA 表达的变化}

\subsection{1 双侧 CPN 损伤}

损伤后 1 周, 3 只鼠中有 2 只的 $\mathrm{CPN}$ 切片 上, 可见中等强度的 B- $50 \mathrm{mRNA}$ 阳性反应细 胞. 亦呈散在性分布 (图 3(a)). 损伤后 3 周, 所 观察的 5 只鼠的 CPN 上,都出现广泛分布的

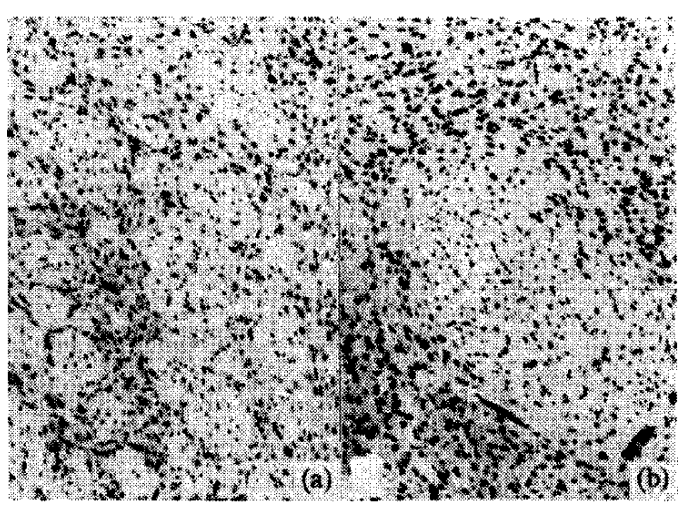

图 2 大鼠尾壳核的尼氏染色 $\times 28$ (a) 生理盐水注射后, (b) 海人酸注射后 
B-50mRNA 强阳性标记信号 (图 3(b)). 至损伤后 6 周, 阳性反应细胞已趋向减 少, 阳性信号水平也比 3 周时减弱, 但仍 高于对照水平 (图 3(c)). B-50mRNA 的 动态变化在损伤的双侧 CPN 内基本相 同.

\subsection{2 单侧 $\mathrm{CPN}$ 损伤}

在损伤侧, B- 50mRNA 标记信号的 分布和强度的变化, 完全类似双侧 CPN 损伤的结果. 最强的杂交信号也是出现 在损伤后 3 周. 对照侧 $\mathrm{CPN}$, 术后 1 周和 6 周均未见 B- $50 \mathrm{mRNA}$ 表达的显著改 变. 只是在术后 3 周, B- $50 \mathrm{mRNA}$ 水平

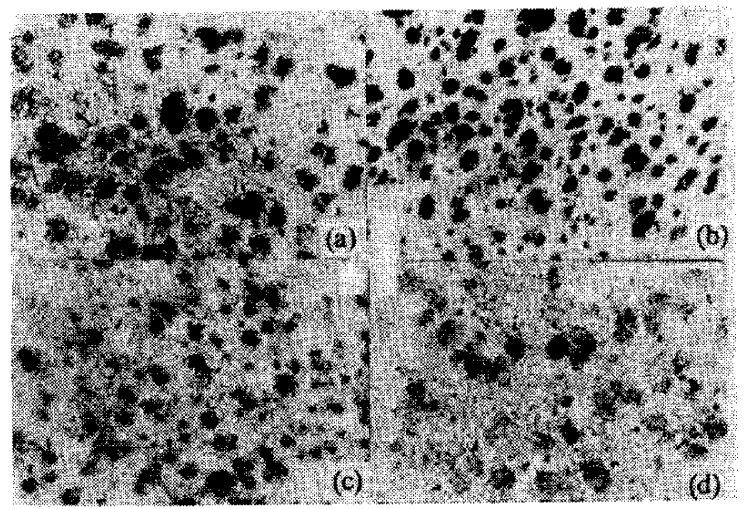

图 3 海人酸损伤后尾壳核内. B $-50 \mathrm{mRNA}$ 的表达 $\times 70$ (a) 损伤后 1 周, (b) 损伤后 3 周, (c) 损伤后 6 周, (d) 正常对照 亦升高, 但仍低于损伤侧水平 (图 4,5).

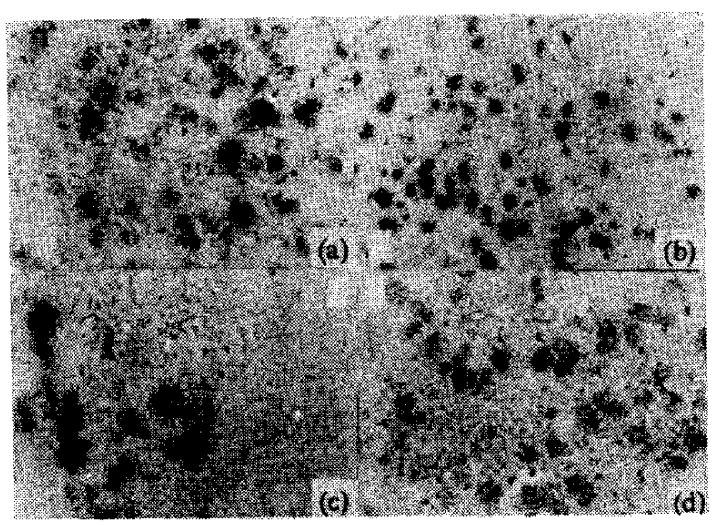

图 4 单侧海人酸损伤的对照侧尾壳核内 B-50mRNA 的表达 $\times 70$

(a) 损伤后 1 周, (b) 损伤后 3 周, (c) 损伤后 6 周, (d) 正常对照

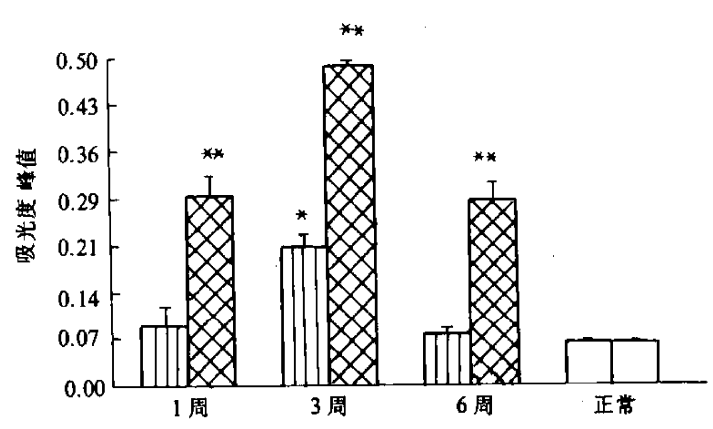

图 5 海人酸损伤单侧尾壳核后 B- $50 \mathrm{mRNA}$ 表达的光密度分析

$\bar{X} \pm \mathrm{SE}$ ，损伤侧， Шा对照侧. * 与对照比较 $p<0.05, * *$ 与对照比较 $p<0.01$

\subsection{3 半定量分析}

光密度扫描结果表明, KA 双侧或单侧注射后 1,3 和 6 周, CPN 内 B-50mRNA 表达的吸 光度峰值都显著地高于对照值. 其最高值比对照值升高 3 10 倍. KA 注射于单侧 CPN 后 光密度扫描结果示于图 5.

\section{3 讨论}

文献报道, 神经生长相关蛋白 B-50 在发育的神经系统内高度表达. 同时在发育成熟后 的若干脑区, 仍可见此蛋白及其 mRNA 持续表达. 本文结果表明, 正常成年大鼠海马锥体细 胞内, B- $50 \mathrm{mRNA}$ 水平比较高, 而在 CPN 内的表达水平较低. 与前人的报道相符. 我们的 主要发现是, 兴奋毒性损伤 CPN 后, 其神经元内 B- $50 \mathrm{mRNA}$ 水平明显升高并持续至 6 周. 
以损伤后 3 周时的表达水平最高,而且高表达的神经元也较多. 以上变化在 CPN 双侧损伤 或单侧损伤都是一致的. 而同一动物另一侧 CPN 注射生理盐水后, B-50mRNA 水平末见明 显变化. 故我们推测上述 B-50mRNA 表达升高的动态过程, 可能是 CPN 内与变性坏死神 经元有纤维联系的, 其轴突末端亦受损伤的神经元的应答反应. 即 B-50 基因转录被激活. 虽然有研究报告某些中枢神经元损伤后, B-50 的合成和转运未见增高 ${ }^{[3]}$, 作者们因而认为这 可能是中枢神经元不能再生的原因之一. 可是另有研究发现一些中枢神经元在其轴突切断 后, 能向上调节其细胞内 B-50mRNA 的水平 ${ }^{[4]}$. 本工作首次报告兴奋毒性损伤中枢神经元 能引起该脑区内 B- $50 \mathrm{mRNA}$ 表达增强. 值得注意的是新近超微结构研究证实, 兴奋毒性损 伤的大鼠 CPN 内, 既有明显的神经元缺失和突触密度减少, 又在损伤后数周至数月内, 出现 突触增生和神经回路重建等再生变化 ${ }^{[9}$. 同时对 Huntington 氏病患者脑病理学检查也观察到 新纹状体内, 变性和再生性变化同时存在 ${ }^{[6]}$. 既然 B-50 被认为是与外周神经再生有密切关系 的一种分子, 这些资料与本文结果联系起来分析, 我们所发现的 CPN 损伤后 B-50mRNA 表 达增强, 可能与 CPN 内存活的神经元某些结构再生有关. 然而文献上亦报告, 某些中枢神经 元损伤后即使 B-50mRNA 高表达,亦不伴随结构再生 ${ }^{[4]}$. 我们设想这是由于中枢神经系统 的高度复杂性,不同类型神经元损伤后的分子、生化和形态的变化可能不尽相同. 加上影响 中枢神经系统再生的内在和外在 (微环境等)因素甚多, 因此有关 B-50 在各种中枢神经元损 伤后的表达变化及意义, 还须深人研究.

致谢承蒙荷兰乌德列支大学 Rudolf Magnus 研究所 J. Verhaagen 博士赠送 B-50cDNA， 特表谢意.

\section{参考文献}

1 Cotman C W, Nieto-Sampedro M. Cell biology of synaptic plasticity. Science, 1984, 225: $1287 \sim 1294$

2 Benowitz L I, Perrone-Bizzozero N I, Neve R L et al. GAP-43 as a marker for structural plasticity in the mature CNS. Prog Brain Res, 1990, 86: 309 320

3 Skene J H P, Willard M. Axonally transported proteins associated with axon growth in rabbit central and peripheral system. J Cell Biol, 1981, 89: $96 \sim 103$

4 Verhaagen J, Zhang Y, Hamers F P T et al. Elevated expression of B-50(GAP-43)-mRNA in a subpopulation of olfactory bulb mitral cells following axotomy. J Neurosci Res, 1993, 35: 162 169

5 Roberts R C, Difiglia M. Evidence for synaptic proliferation reorganization and growth in the exitotoxic lesioned adult rat caudate nucleus. Exp Neurol, 1990, 107: 1 10

6 Graveland G A, Williams R S, Difiglia M. Evidence for degenerative and regenerative changes in neostriatal spiny neurons in Hnutington's disease. Scienœ, 1985, 227: 770 773 